\title{
فاعلية اختبار في التمييز بين الطالبة الموهوبين والعاديين على القدرة (الرياضيات) في المرحلة الأساسية الدنيا في الأردن
}

\author{
إعــــــاد \\ أ. سميرعبد|لكريم أحمد الريماويك \\ ماجستير موهبة و إبداع

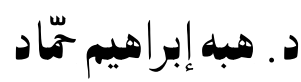 \\ دكتوراه قياس و تقويم
}

مجلة بحوث التربية النوعية - جامعة المنصورة

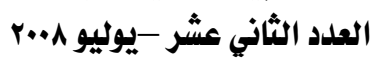




\section{فاعلية اختبار في التهييز بين الطلبة الموهوبين و العاديين على القدرة (الرياضيات) في المرحلة الأساسية الدنيا في الأردن}

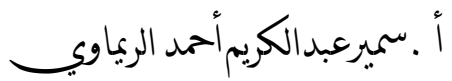

$$
\text { د ـ مبه إبراهيمحمَاد }
$$

\section{ملخحص}

لأن الكثف و التعامل مـع الطلبـة الموهوبين أمرا هاما لما تمثله هذه الفئة من أهمية يِّ نجاح

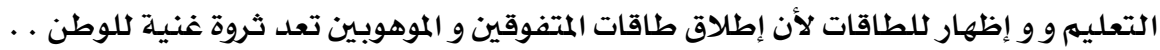

$$
\text { هدف البحث إلى الإجابة على السؤال التالي : }
$$

ما الفاعلية التمييزية لاختبار يميز بين الطلبـة العاديين و الموهوبين يِ القدرة الرياضية

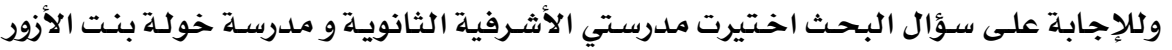

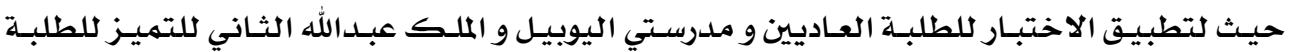

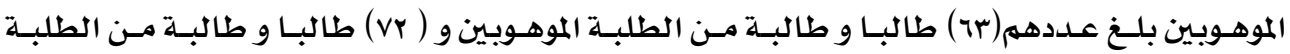

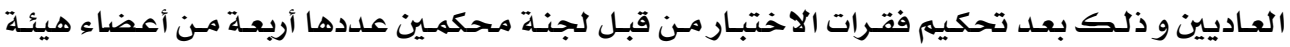

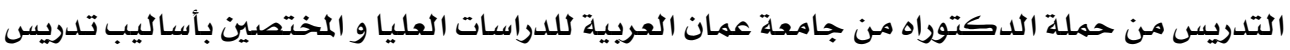

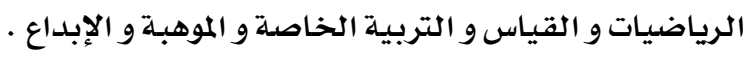

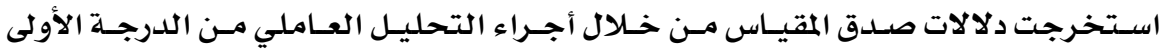

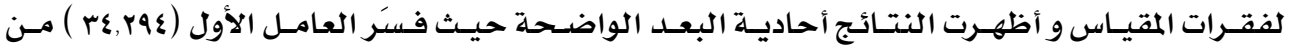

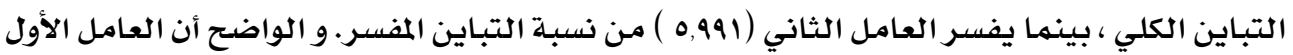

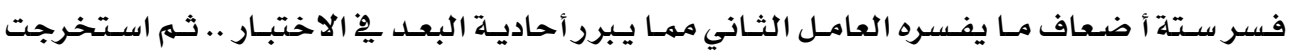

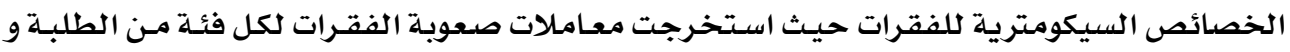

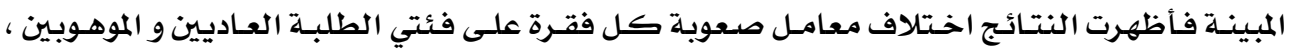

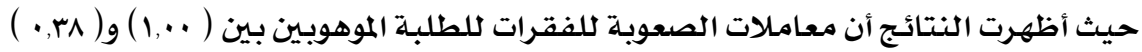

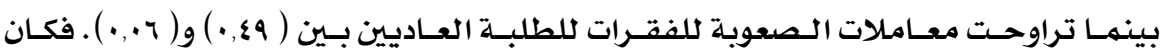

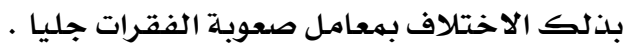
وباسـتخـراج معامـل التمييـز للفقـرات للمهجمـوعتين أظهـرت النتــائج أن معـاملات التمييـز

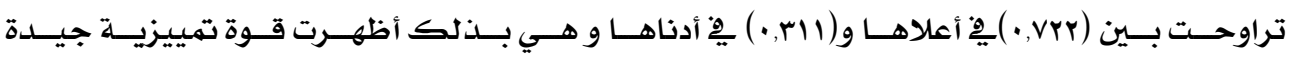

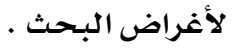
ويإجراء تحليـل التبـاين الأحسادي للكشف عن قدرة الاختبـار على التمييز بـين فئتي الطلبـة

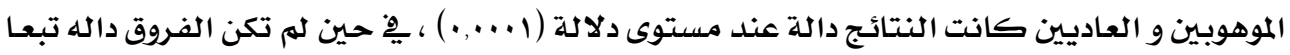




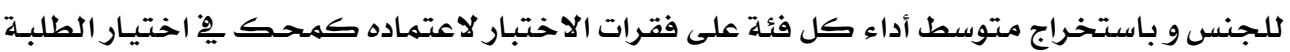

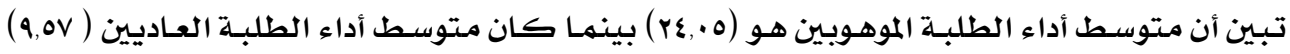

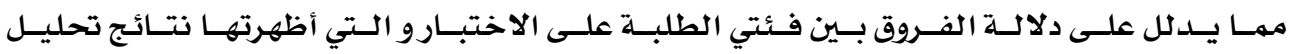

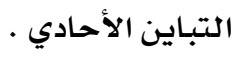

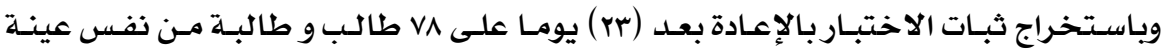

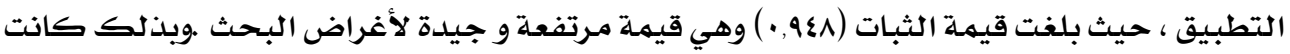

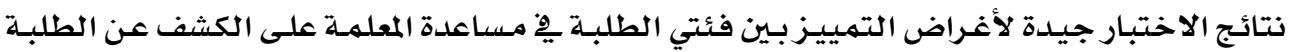

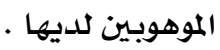

ومن هنا تظهر الكثف و التعامل مـع الطلبة الموهوبين لديهم لما تمثلـه هـذه الفئسة مـن أهميـة

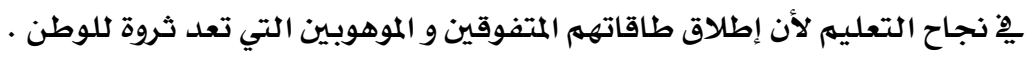

\section{الاقدمة و أهمية البهث}

اهتمت المجتمعات المختلفة منذ القدم بالتعرف على الموهوبين من أبنائها ، مثل الولايات

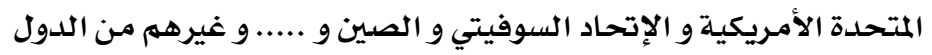

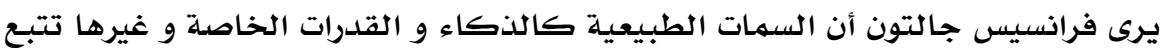

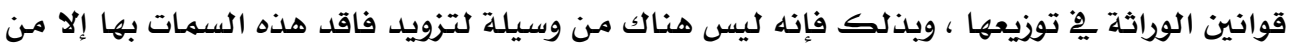

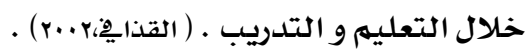

كما يرى العلماء أن وراثة تلك السمات لا تتدخل ِِّ تحديد الحدود القصوى و الدنيا التي

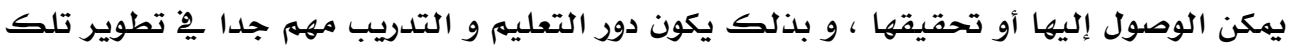

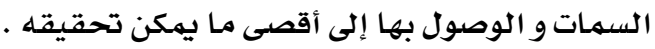

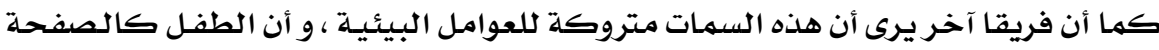

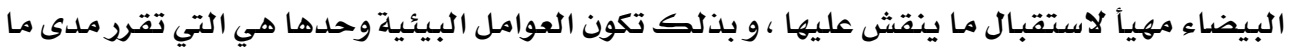

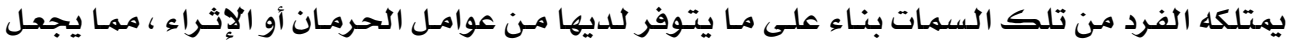

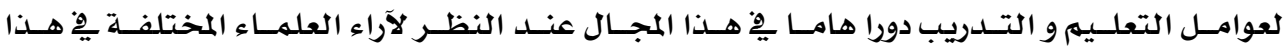

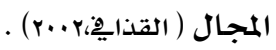

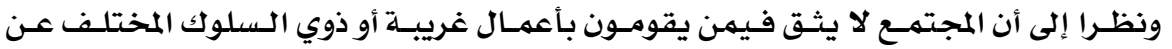

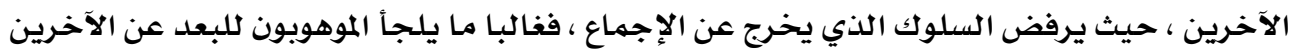

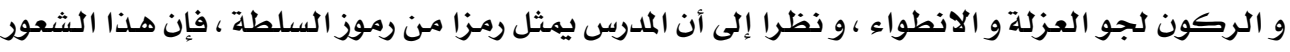

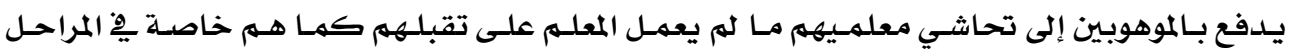

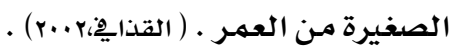

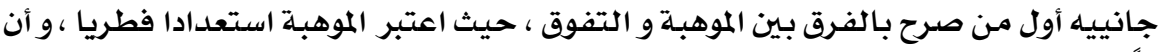

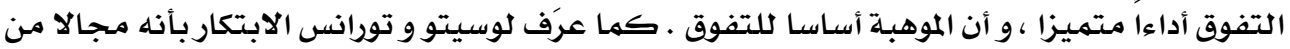




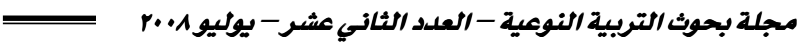

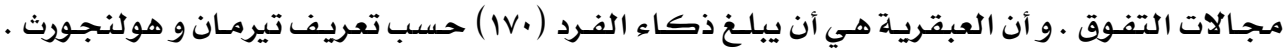

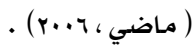

عرف هيلدريث الطفل المتفوق بأنه طفل الموهوب سواء كانت موهبته يْ مـجـال أكاديهي أو

$$
\text { مجال آخر مثل الموسيقى و الرسهم و التمثيل (ماضي ، ج +. r) . }
$$

ويرى رنزولي Renzolli أن الأطفال الموهوبين هم الذين لديهم القدرة على إظهار أو تحقيق

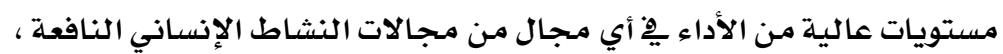

كما أوضـح ِِّ نهوذجه الثلاثي الحلقات أن الموهبة تتألف مـن ناتج تفاعل ثلاث مجموعات

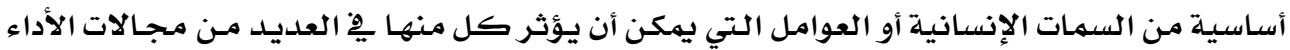

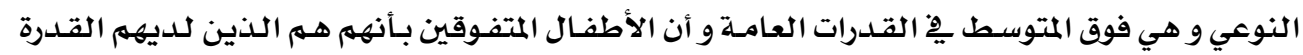

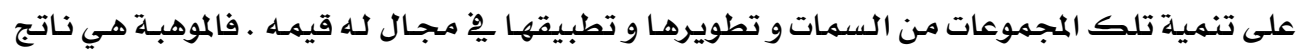
تقاطع الحلقات الثلاث ، و التفوق هو القدرة على تطـوير و تطبيق تلـك المجموعات مـن السمات التي تشملها الحلقات . ( القريطي ، ا... ) .

ويـرى القريطي أن الموهبـة تهثل القـــرات و الاسـتعدادات الفطريـة لـدى الفـرد لكي يتفـوق و

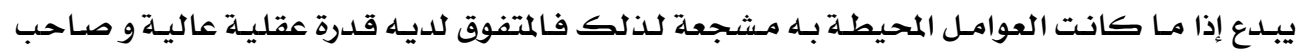

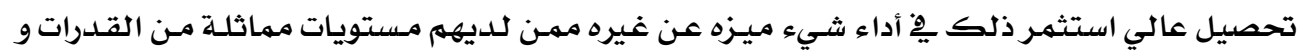
الاستعدادات ، و المبدع هو موهوب و مـتفوق عمـن حوله ، و العبقري شخص تريـع على قهـة التفـوق و هـو

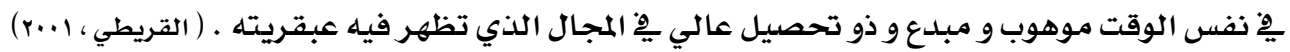
وكهـا يـرى المختصون مـثل عبــ السـلام عبــ الغفـار و يوسـف الشيخ أن التعـرف المبكر على

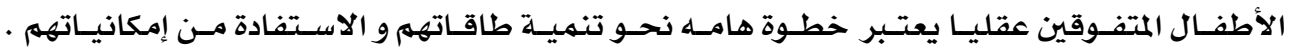

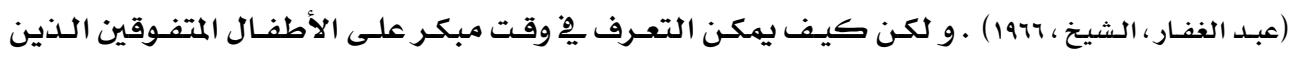

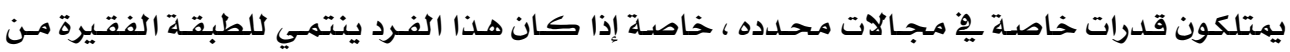

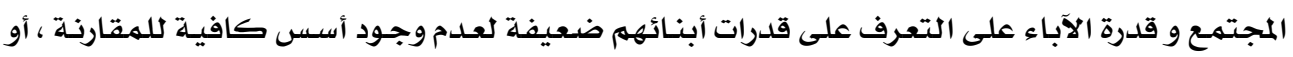

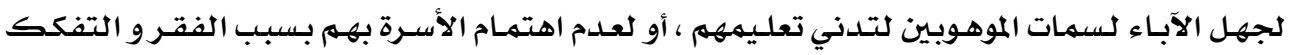
الأسـري التي تعاني منـه عادة هذه الفئة مـن المجتمعات .

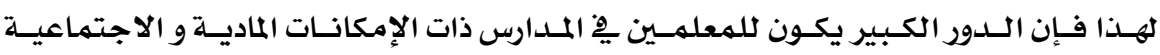

الضعيفة فِ اكتشاف الطلبة الموهوبين لديهم بكل الطرق المتاحة لهم كل حسب الجـانب الذي يتفوق بله ، لذلك كان لا بـ من أن يهتم المعلم بأكثر من طريقة لجمع المعلومات عن الطالب الـذي يشك

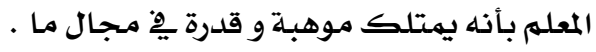

وبتــوع أسـاليب الكسشف عـن الموهـوبـين مـن خـلال : اختبــارات الـذكاء ، مقـاييس التقـــير

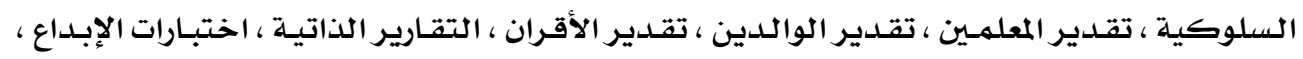

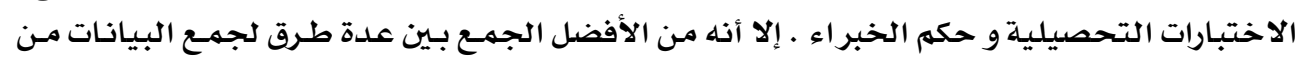




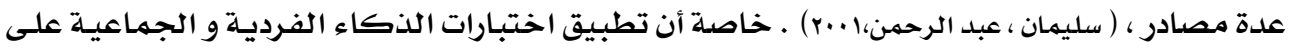

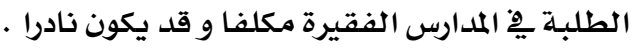

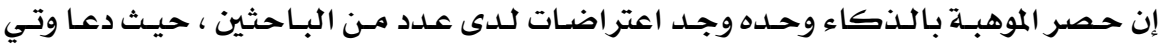

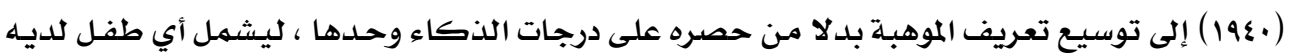

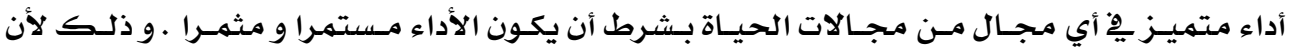

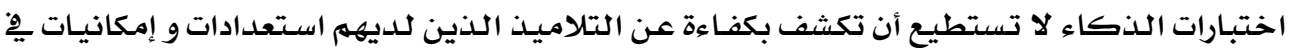

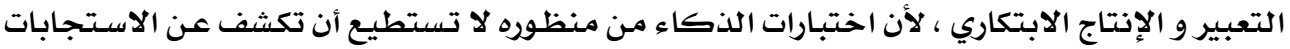

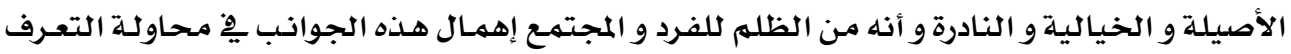

على الموهوبين (Greenlaw \& Mclontosh,1988)

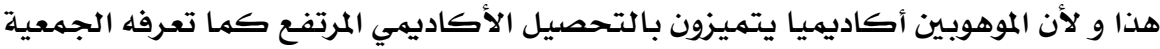

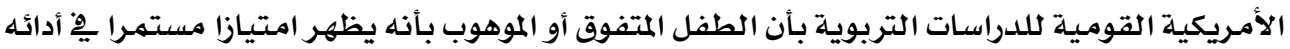

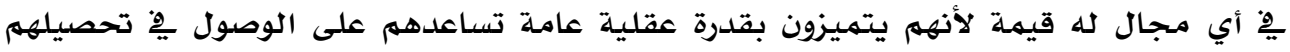

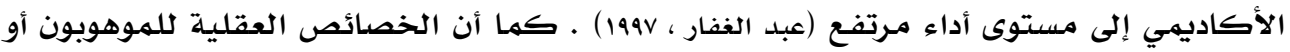
المتفوقون أكاديميا واضحلة و يسهل تمييزها مثل :

- - تمتع الموهوبون بمقدرة عقلية عالية تظهر على شكل أداء مـرتفع على اختبـارات الذكاء

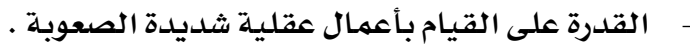

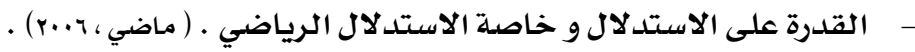

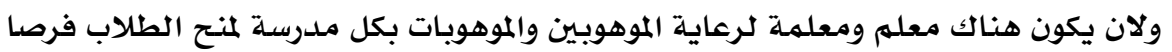

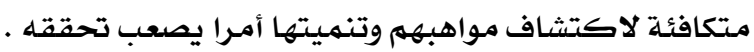

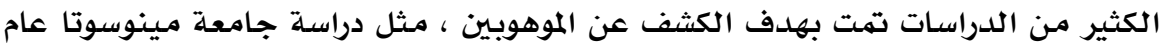

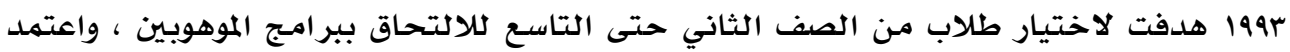

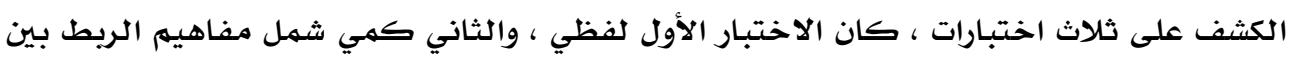

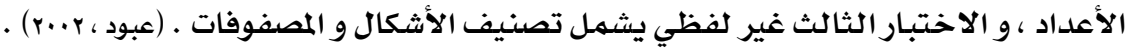

وكـذلك دراسـة أجريست على البيئـة العربيـة ، قـام بهـا النيلـي و الصمادي و جـلال (1997 )

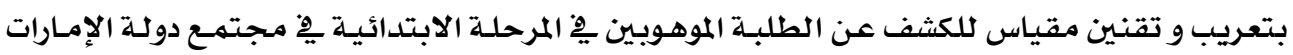

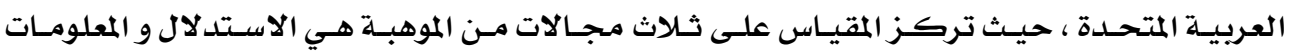

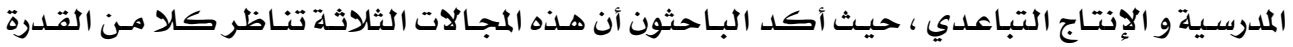

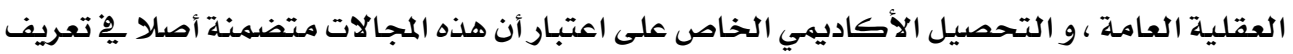

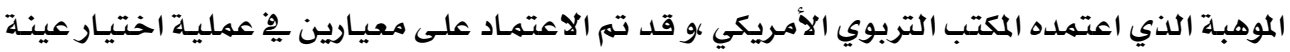

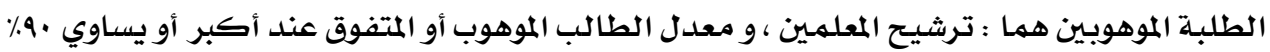




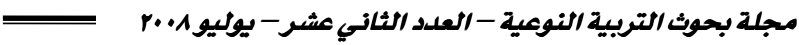

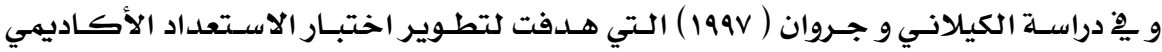

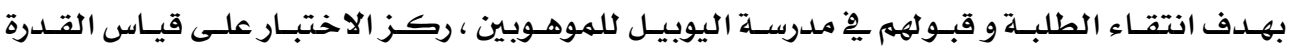
اللفظية و القدرة الرياضيـة و القدرة على التفكير المنطقي .

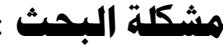

لأن اهتهـام وزارة التربيـة والتعليهم بـالمتفوقين ، يـأتي ضـهـن حلقـة محكمهـة ثـاملـة، تنبثق مـن المنطلقات الآتية :

- تطوير النظام التربوي وزيادة كفاءته بالاعتماد على تطوير واستحداث برامـج أكثر فاعليـة.

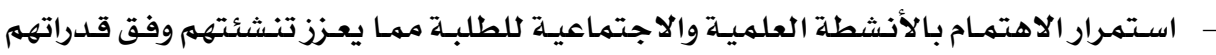
العقلية وكفاءاتهم ومتطلبات تعليمهم وتنهيلة شخصياتهم . - تنميلة شخصية الطالب المتفوق من جميع الجوانب بصورة متوازنة متكاملة .

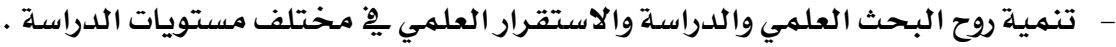

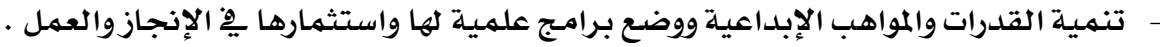
ولأن عمليـة الكثف عن الطلبـة المتفـوقين عقليـا مـن الأمـور الهامـة فهـن خلالهـا يـتم التعـرف على مـلدخلات التعلـيهم و اسـتعداداتهم و تحديـد متطلبـاتهم و حاجـاتهمه ، و هـذه العمليـة هـي مـن أهـم عواهل نجاح التعليهم و رعاية الموهوبين و المتفوقين و إطلاق طاقاتهم .

ويذكر بـدر العمـر عدة مسلـمات يجبـ أن تؤخذ بعين الاعتبـار عند التعـرف على المتفوقين :

ا - - من يتم وصفهم بأنهم متفوقين قد لا يتفوقون على الآخرين يْ جميع التخصصات .

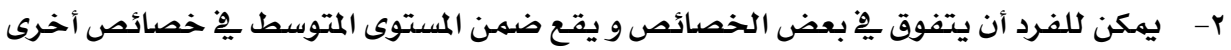

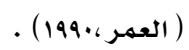

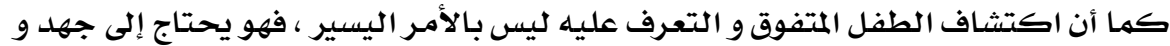
رؤيسة و بصيرة يِ اختيار الطريقة و الأسلوب المناسب، لأن الطرق ألمستخلدمه تختلف باختلاف التعريف الـذي يـتم اعتهــاده ـو يفـضل الاعتمـاد على أكثر مـن طريقـة ليـتم اسـتثمار مزايـا الطـرق و تـلايف السلبيات فيها السيات

ويخ تعريف فليجروبث (1909) للمتفوقين عقليا بأنهم من يصلون يخ تحصيلهم الأكاديهي

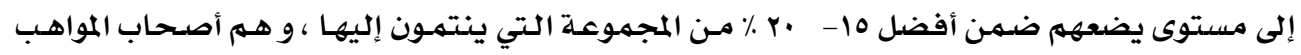

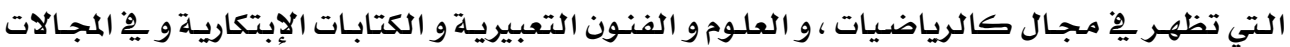

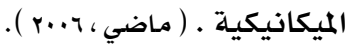

إن الطالب المتفوق عقليا يتميز بصفات و خصائص لا توجد يْ غيره ، لذلك لا بـ من وجود منهج يتميز بـالاختالاف النوعي ، و لأن مادة الرياضيات تمتازبالتجريد و هي أيضا مـادة نشطة ، حيـث يمكن إدرالك الحقـائق و اسـتيعابها ثم الانتقـال منهـا إلى معلومـات أخـرى ، و هـي مـادة حيويـة و حقل 
خصب لاستمـرارية بناء التفكير لدى الطلبة ـ كانت جميع اختبارات و مقاييس الكثف عن الموهوبين

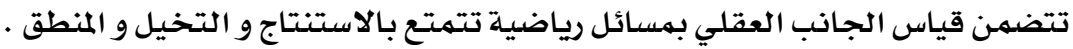

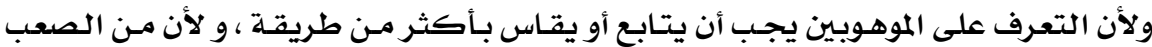

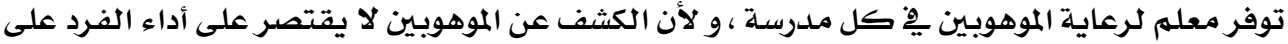

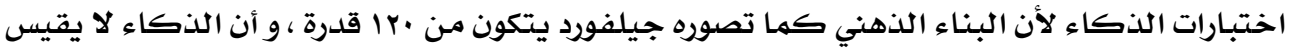

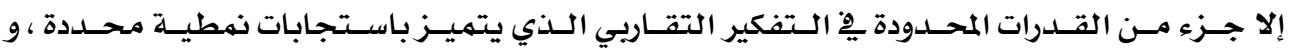

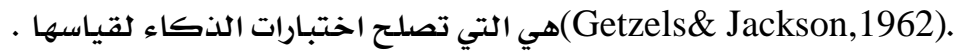

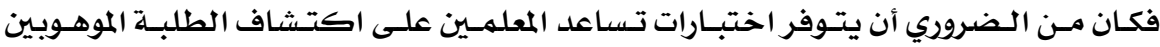

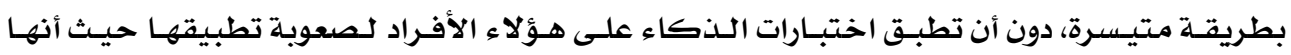

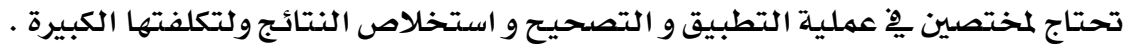

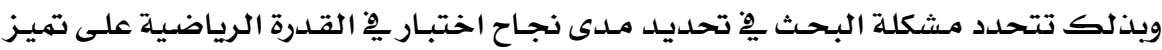

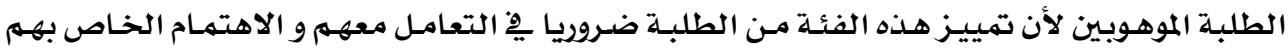

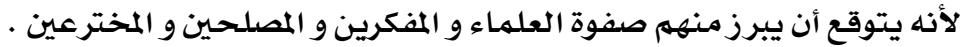

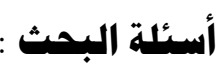

$$
\text { بالتحديد يجيب البحث عن السؤال التالي : }
$$

1- ما الفاعلية التمييزية لاختبار القدرة الرياضيلة يِّ التمييز بين الطلبة العاديين و الموهوبين ؟

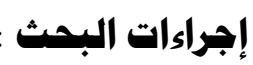

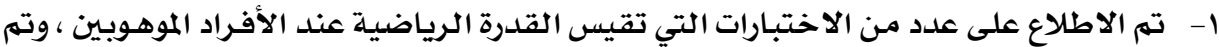

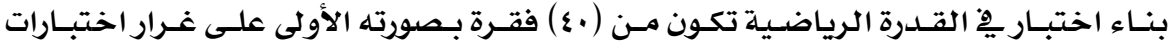

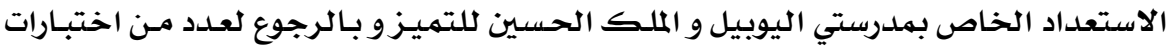

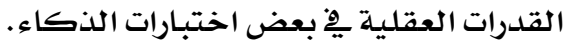

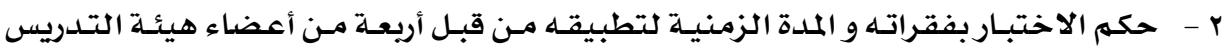

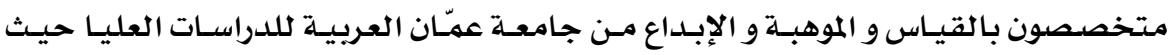

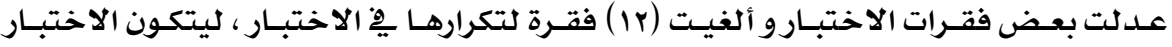

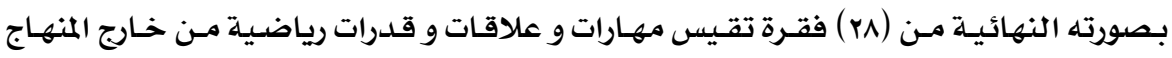

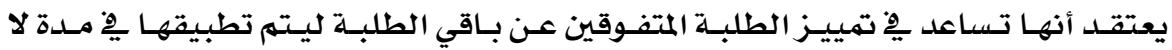
تتعدى 0ـ دقيقة . r - ولاستخراج دلالات الصدق و الثبات طبق اختبار القدرة الرياضية على VV طالبـا و مه طالبـة

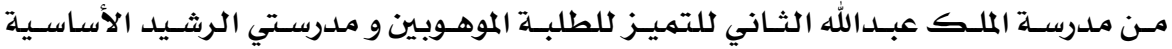

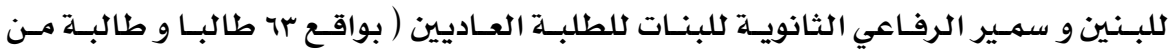

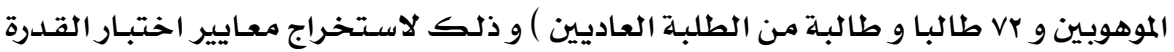




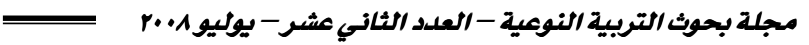

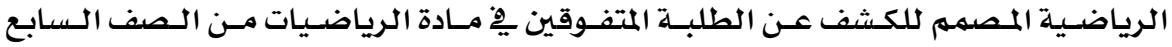

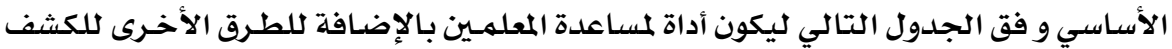
عن الموهوبين.

جدول (1) عينة تطبيق اختبار القدرة الرياضية

\begin{tabular}{|c|c|c|c|}
\hline المجموع & طلبـة عاديين & طلبـة متفوقين & \\
\hline VV & $\varepsilon$. & rv & ذكور \\
\hline $0 \wedge$ & rr & Y & إناث \\
\hline iro & Vr & זד & مجمهوع \\
\hline
\end{tabular}

دلات صدق الاختبار :

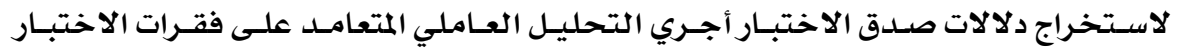

فكانت النتائج كالتالي : 


\begin{tabular}{|c|c|c|c|}
\hline ل الاختبار & ل مر للعوا & ور الكامنة و نسب الت & جدول(r) يوضح قي \\
\hline \multirow{2}{*}{ العوامل } & \multicolumn{3}{|c|}{ قيم الجذور الكامنة } \\
\hline & الكلي & نسبـة التبـاين المفسنر & Cumulative $\%$ \\
\hline 1 & 9.602 & 34.294 & 34.294 \\
\hline 2 & 1.677 & 5.991 & 40.285 \\
\hline 3 & 1.441 & 5.147 & 45.431 \\
\hline 4 & 1.361 & 4.860 & 50.291 \\
\hline 5 & 1.300 & 4.643 & 54.934 \\
\hline 6 & 1.064 & 3.800 & 58.734 \\
\hline 7 & .989 & 3.532 & 62.266 \\
\hline 8 & .915 & 3.266 & 65.532 \\
\hline 9 & .900 & 3.213 & 68.745 \\
\hline 10 & .841 & 3.003 & 71.749 \\
\hline 11 & .773 & 2.760 & 74.508 \\
\hline 12 & .723 & 2.582 & 77.091 \\
\hline 13 & .713 & 2.545 & 79.636 \\
\hline 14 & .604 & 2.158 & 81.793 \\
\hline 15 & .554 & 1.977 & 83.770 \\
\hline 16 & .527 & 1.881 & 85.651 \\
\hline 17 & .520 & 1.859 & 87.510 \\
\hline 18 & .471 & 1.684 & 89.193 \\
\hline 19 & .445 & 1.590 & 90.783 \\
\hline 20 & .412 & 1.471 & 92.254 \\
\hline 21 & .378 & 1.349 & 93.602 \\
\hline 22 & .354 & 1.263 & 94.866 \\
\hline 23 & .321 & 1.145 & 96.011 \\
\hline 24 & .287 & 1.024 & 97.034 \\
\hline 25 & .259 & .924 & 97.958 \\
\hline 26 & .208 & .742 & 98.700 \\
\hline 27 & .188 & .671 & 99.371 \\
\hline 28 & .176 & .629 & 100.000 \\
\hline
\end{tabular}

Extraction Method: Principal Component Analysis.

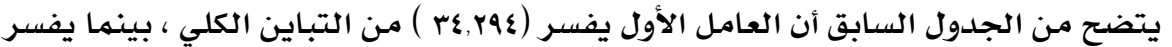

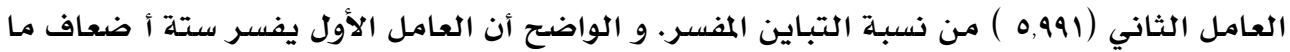




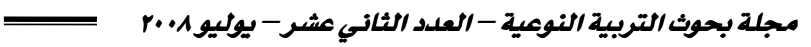

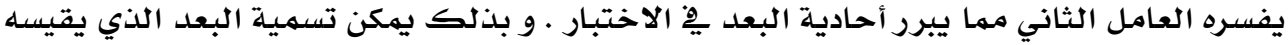

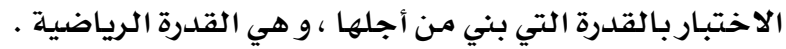

كما تلاحظ أحادية البعد فِ الرسهم البياني للتحليل العاملي كالتالي : الشكل (1) يمثل أحادية البعد ِِ الرسم البياني للتحليل العاملي :

\section{Scree Plot}

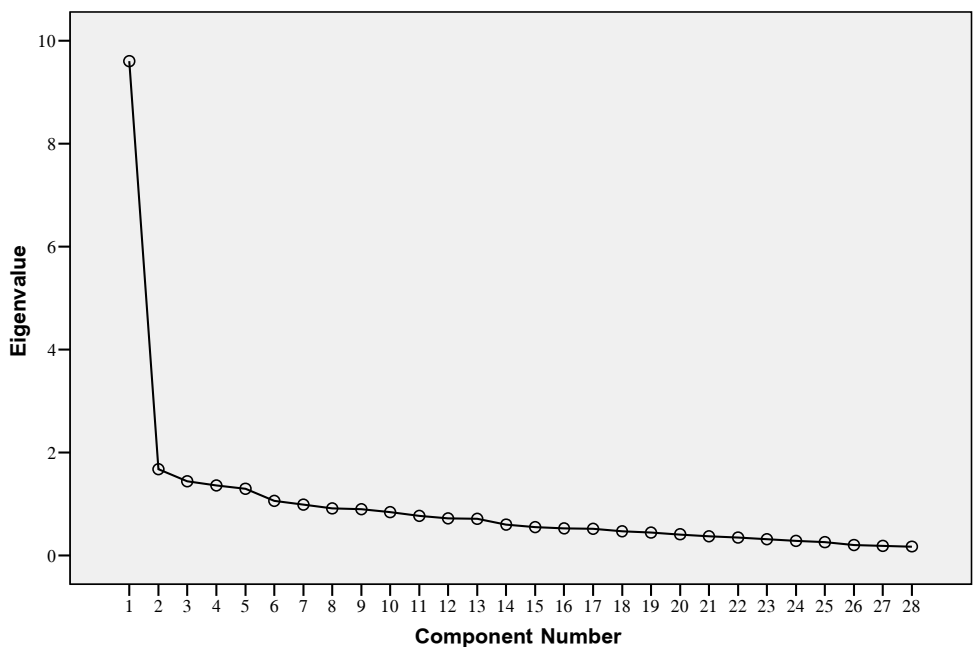

وباستخراج معامل صعوبـة الفقرات لكل مـن الطلبـة الموهوبين و العاديين كانت النتائج كما يوضحها الجدولين التاليين : 
جدول (r) يمثل صعوبة الفقرات للطلبة الموهوبين

\begin{tabular}{|c|c|c|c|}
\hline & المجموع & العدد & صعوبة الفقرات \\
\hline Q1 & 58 & 63 & .92 \\
\hline Q2 & 54 & 63 & .86 \\
\hline Q3 & 59 & 63 & .94 \\
\hline Q4 & 56 & 63 & .89 \\
\hline Q5 & 43 & 63 & .68 \\
\hline Q6 & 60 & 63 & .95 \\
\hline Q7 & 60 & 63 & .95 \\
\hline Q8 & 62 & 63 & .98 \\
\hline Q9 & 59 & 63 & .94 \\
\hline Q10 & 48 & 63 & .76 \\
\hline Q11 & 57 & 63 & .90 \\
\hline Q12 & 48 & 63 & .76 \\
\hline Q13 & 33 & 63 & .52 \\
\hline Q14 & 58 & 63 & .92 \\
\hline Q15 & 60 & 63 & .95 \\
\hline Q16 & 59 & 63 & .94 \\
\hline Q17 & 52 & 63 & .83 \\
\hline Q18 & 59 & 63 & .94 \\
\hline Q19 & 57 & 63 & .90 \\
\hline Q20 & 54 & 63 & .86 \\
\hline Q21 & 63 & 63 & 1.00 \\
\hline Q22 & 63 & 63 & 1.00 \\
\hline Q23 & 57 & 63 & .90 \\
\hline Q24 & 61 & 63 & .97 \\
\hline $\mathrm{Q} 25$ & 45 & 63 & .71 \\
\hline Q26 & 62 & 63 & .98 \\
\hline Q27 & 24 & 63 & .38 \\
\hline Q28 & 44 & 63 & .70 \\
\hline MAX & 1515 & 63 & 24.05 \\
\hline
\end{tabular}




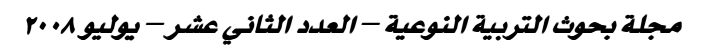

جدول (ع) صعوبة الفقرات للطلبة العاديين

\begin{tabular}{|c|c|c|c|}
\hline & المجموع & العدد & صعوبة الفقرة \\
\hline Q1 & 25 & 72 & .35 \\
\hline Q2 & 29 & 72 & .40 \\
\hline Q3 & 29 & 72 & .40 \\
\hline Q4 & 31 & 72 & .43 \\
\hline Q5 & 26 & 72 & .36 \\
\hline Q6 & 31 & 72 & .43 \\
\hline Q7 & 25 & 72 & .35 \\
\hline Q8 & 35 & 72 & .49 \\
\hline Q9 & 31 & 72 & .43 \\
\hline Q10 & 25 & 72 & .35 \\
\hline Q11 & 15 & 72 & .21 \\
\hline Q12 & 16 & 72 & .22 \\
\hline Q13 & 15 & 72 & .21 \\
\hline Q14 & 20 & 72 & .28 \\
\hline Q15 & 24 & 72 & .33 \\
\hline Q16 & 24 & 72 & .33 \\
\hline Q17 & 21 & 72 & .29 \\
\hline Q18 & 20 & 72 & .28 \\
\hline Q19 & 25 & 72 & .35 \\
\hline Q20 & 28 & 72 & .39 \\
\hline $\mathrm{Q} 21$ & 22 & 72 & .31 \\
\hline $\mathrm{Q} 22$ & 25 & 72 & .35 \\
\hline Q23 & 26 & 72 & .36 \\
\hline Q24 & 28 & 72 & .39 \\
\hline Q25 & 27 & 72 & .38 \\
\hline Q26 & 28 & 72 & .39 \\
\hline Q27 & 4 & 72 & $5.56 \mathrm{E}-02$ \\
\hline Q28 & 34 & 72 & .47 \\
\hline MAX & 689 & 72 & 9.57 \\
\hline
\end{tabular}




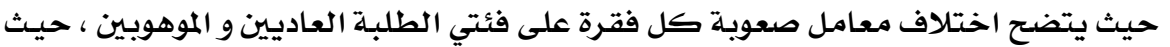

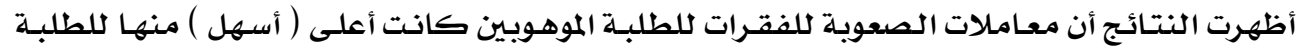

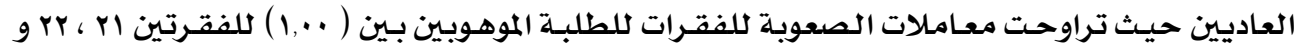

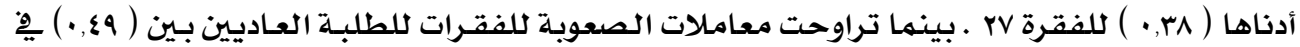

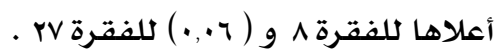

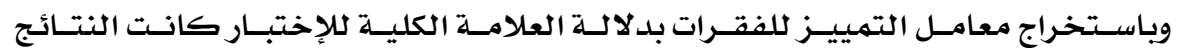
كالتالي :

جدول (ه) معاملات التمييز لفقرات اختبار القدرة الرياضية

\begin{tabular}{|c|c|c|}
\hline الفقرات & & معامل الالتمييز \\
\hline \multirow[t]{2}{*}{$\mathrm{q} 1$} & Pearson Correlation & $.628(* *)$ \\
\hline & Sig. (2-tailed) & .000 \\
\hline \multirow[t]{2}{*}{$\mathrm{q} 2$} & Pearson Correlation & $.517(* *)$ \\
\hline & Sig. (2-tailed) & .000 \\
\hline \multirow[t]{2}{*}{$\mathrm{q} 3$} & Pearson Correlation & $.614(* *)$ \\
\hline & Sig. (2-tailed) & .000 \\
\hline \multirow[t]{2}{*}{$\mathrm{q} 4$} & Pearson Correlation & $.465(* *)$ \\
\hline & Sig. (2-tailed) & .000 \\
\hline \multirow[t]{2}{*}{$\mathrm{q} 5$} & Pearson Correlation & $.353(* *)$ \\
\hline & Sig. (2-tailed) & .000 \\
\hline \multirow[t]{2}{*}{ q6 } & Pearson Correlation & $.548(* *)$ \\
\hline & Sig. (2-tailed) & .000 \\
\hline \multirow[t]{2}{*}{$\mathrm{q} 7$} & Pearson Correlation & $.661(* *)$ \\
\hline & Sig. (2-tailed) & .000 \\
\hline \multirow[t]{2}{*}{ q8 } & Pearson Correlation & $.595(* *)$ \\
\hline & Sig. (2-tailed) & .000 \\
\hline \multirow[t]{2}{*}{ q9 } & Pearson Correlation & $.536(* *)$ \\
\hline & Sig. (2-tailed) & .000 \\
\hline \multirow[t]{2}{*}{$q 10$} & Pearson Correlation & $.475(* *)$ \\
\hline & Sig. (2-tailed) & .000 \\
\hline \multirow[t]{2}{*}{$\mathrm{q} 11$} & Pearson Correlation & $.711(* *)$ \\
\hline & Sig. (2-tailed) & .000 \\
\hline \multirow[t]{2}{*}{$\mathrm{q} 12$} & Pearson Correlation & $.587(* *)$ \\
\hline & Sig. (2-tailed) & .000 \\
\hline q13 & Pearson Correlation & $.409(* *)$ \\
\hline
\end{tabular}




\begin{tabular}{|c|c|c|}
\hline \multicolumn{2}{|c|}{ 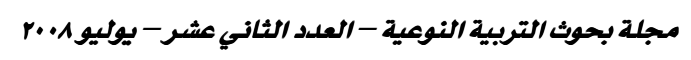 } & \multirow{2}{*}{ معامل التتمييز } \\
\hline الفقرات & & \\
\hline & Sig. (2-tailed) & 0.000 \\
\hline \multirow{2}{*}{$\mathrm{q} 14$} & Pearson Correlation & $.689(* *)$ \\
\hline & Sig. (2-tailed) & .000 \\
\hline \multirow[t]{2}{*}{$\mathrm{q} 15$} & Pearson Correlation & $.625(* *)$ \\
\hline & Sig. (2-tailed) & .000 \\
\hline \multirow[t]{2}{*}{ q16 } & Pearson Correlation & $.649(* *)$ \\
\hline & Sig. (2-tailed) & .000 \\
\hline \multirow[t]{2}{*}{$\mathrm{q} 17$} & Pearson Correlation & $.567(* *)$ \\
\hline & Sig. (2-tailed) & .000 \\
\hline \multirow[t]{2}{*}{ q18 } & Pearson Correlation & $.677(* *)$ \\
\hline & Sig. (2-tailed) & .000 \\
\hline \multirow[t]{2}{*}{ q19 } & Pearson Correlation & $.618(* *)$ \\
\hline & Sig. (2-tailed) & .000 \\
\hline \multirow[t]{2}{*}{$\mathrm{q} 20$} & Pearson Correlation & $.571(* *)$ \\
\hline & Sig. (2-tailed) & .000 \\
\hline \multirow[t]{2}{*}{$\mathrm{q} 21$} & Pearson Correlation & $.709(* *)$ \\
\hline & Sig. (2-tailed) & .000 \\
\hline \multirow[t]{2}{*}{$\mathrm{q} 22$} & Pearson Correlation & $.722(* *)$ \\
\hline & Sig. (2-tailed) & .000 \\
\hline \multirow[t]{2}{*}{$\mathrm{q} 23$} & Pearson Correlation & $.618(* *)$ \\
\hline & Sig. (2-tailed) & .000 \\
\hline \multirow[t]{2}{*}{$\mathrm{q} 24$} & Pearson Correlation & $.679(* *)$ \\
\hline & Sig. (2-tailed) & .000 \\
\hline \multirow[t]{2}{*}{$\mathrm{q} 25$} & Pearson Correlation & $.356(* *)$ \\
\hline & Sig. (2-tailed) & .000 \\
\hline \multirow[t]{2}{*}{$\mathrm{q} 26$} & Pearson Correlation & $.708(* *)$ \\
\hline & Sig. (2-tailed) & .000 \\
\hline \multirow[t]{2}{*}{ q27 } & Pearson Correlation & $.441(* *)$ \\
\hline & Sig. (2-tailed) & .000 \\
\hline \multirow[t]{2}{*}{$\mathrm{q} 28$} & Pearson Correlation & $.311(* *)$ \\
\hline & Sig. (2-tailed) & .000 \\
\hline
\end{tabular}

** Correlation is significant at the 0.01 level (2-tailed).

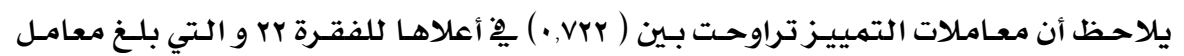

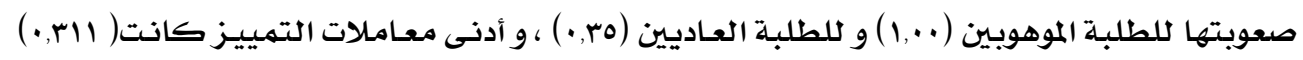




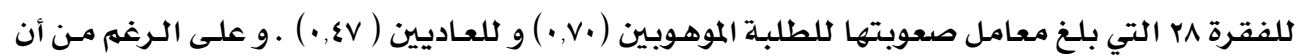

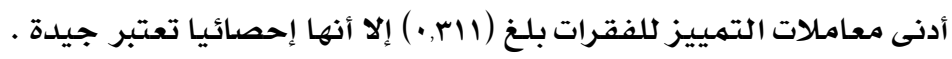
وبإجراء تحليل التباين الأحادي لبيان الفروق بين متوسط أداء الطلبـة الموهـوبين و العـاديين

على فقرات الاختبار تبين ما يلي : عليل التياين

ANOVA

جدول (7) يمثل تحليل التباين الأحادي للفروق بين متوسطات تبعا للجنس و نوع الطلبة

\begin{tabular}{|c|c|c|c|c|c|}
\hline المصلدر & مجمـوع المريعات & درجات الحـرية & متتوسط المربعات & قيمـة ف & الدلالية. \\
\hline الجنس & 3.219 & 1 & 3.219 & .415 & .520 \\
\hline النوع & 6995.094 & 1 & 6995.094 & 902.543 & .000 \\
\hline الجنس التفل بين النوع & 16.997 & 1 & 16.997 & 2.193 & .141 \\
\hline الخطأ & 1015.306 & 131 & 7.750 & & \\
\hline الكلي1 & 44060.000 & 135 & & & \\
\hline $\begin{array}{c}\text { Corrected } \\
\text { Total }\end{array}$ & 8077.659 & 134 & & & \\
\hline
\end{tabular}

يتضـح من الجدول السابق أن هناك فروق بـين متتوسط أداء الطلبـة الموهوبين و متوسط أداء

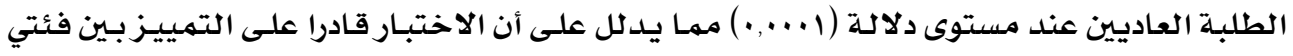

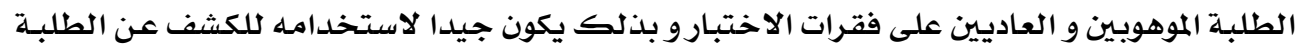

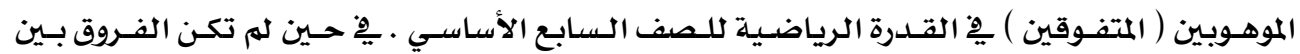

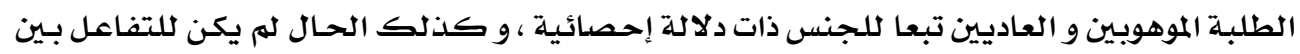

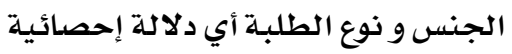

وباستخراج متوسطات الأداء لمجموعتي الطلبة ( العاديين و الموهوبين ) على الاختبـار كانت

النتائج كالتالي : 


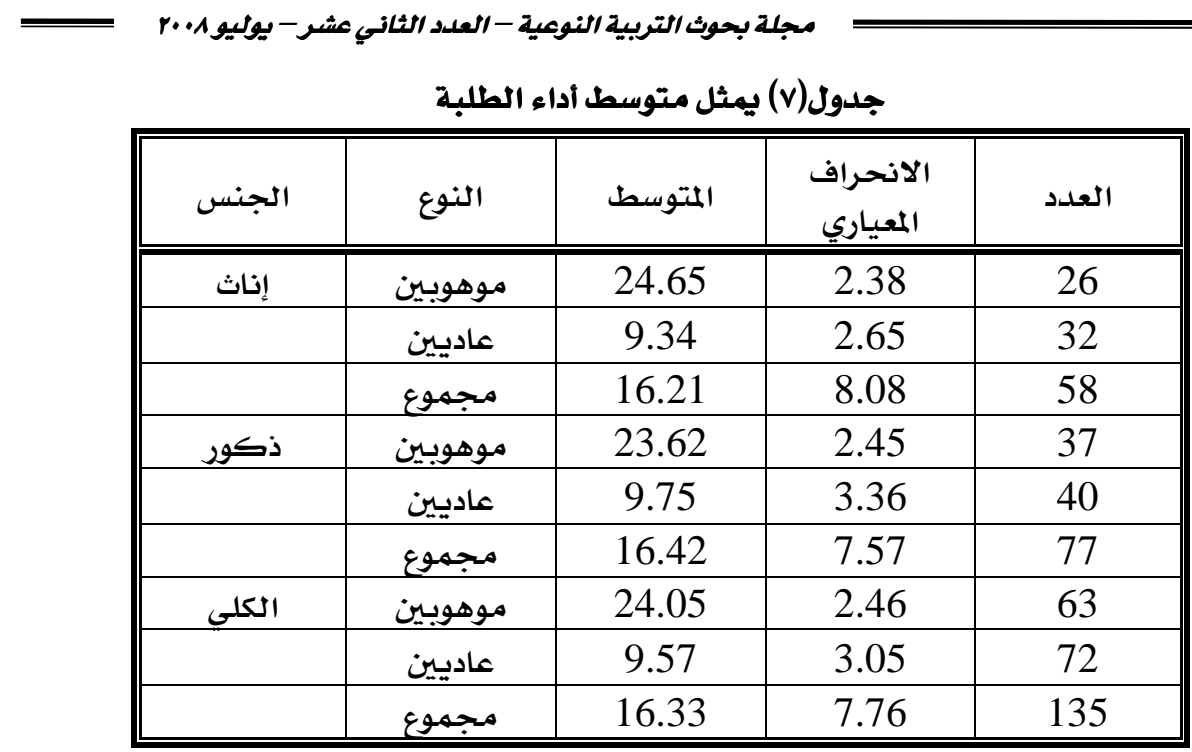

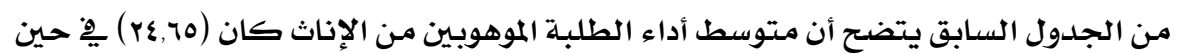

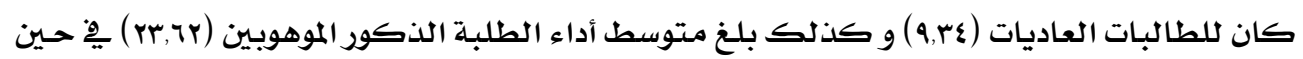

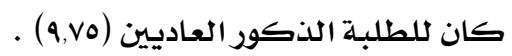

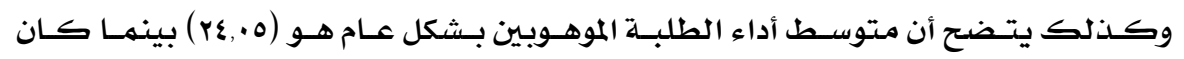

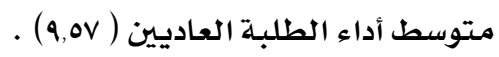
ثبات الاختبار :

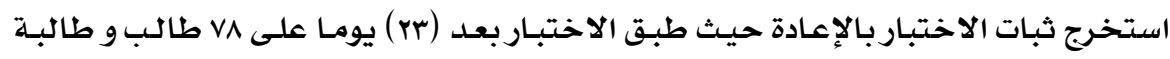

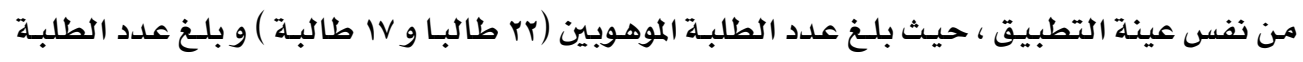

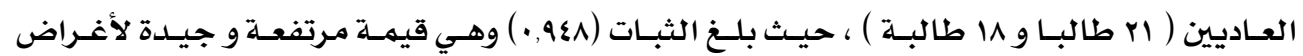

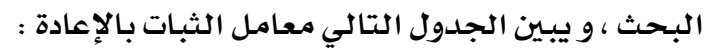

جدول (A) يمثل معامل ثبات الاختبار

\begin{tabular}{|c|c|c|c|}
\hline & & التطبيق الأول & التطبيق الثاني \\
\hline \multirow[t]{3}{*}{ التطبيق الأول } & Pearson Correlation & 1 & $.948(* *)$ \\
\hline & Sig. (2-tailed) & & .000 \\
\hline & $\mathrm{N}$ & 78 & 78 \\
\hline \multirow[t]{3}{*}{ التطبيق الثاني } & Pearson Correlation & $.948(* *)$ & 1 \\
\hline & Sig. (2-tailed) & .000 & \\
\hline & $\mathrm{N}$ & 78 & 78 \\
\hline
\end{tabular}

** Correlation is significant at the 0.01 level (2-tailed). 
-

ما الفاعلية التمييزية لاختبار القدرة الرياضية يِّإنتييز بين الطلبة العاديين و الموهوبين ؟

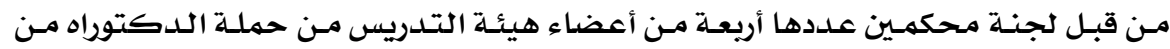

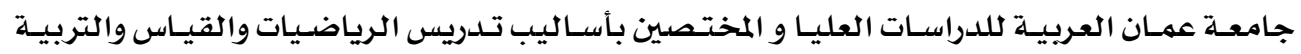

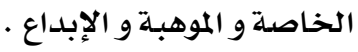

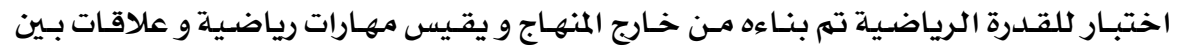

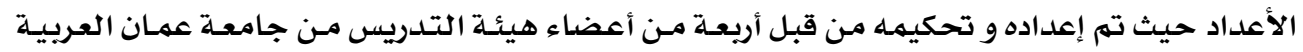

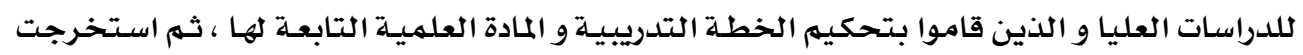

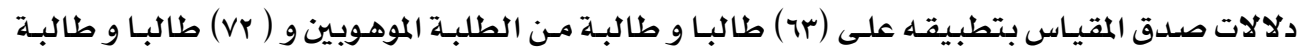

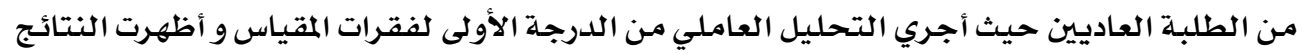

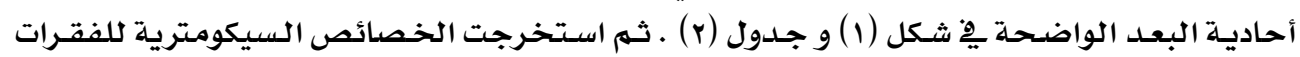

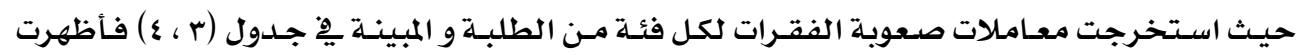

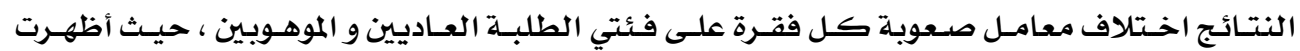

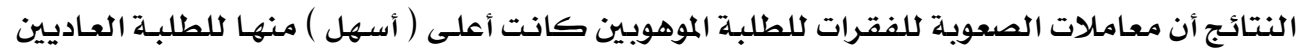

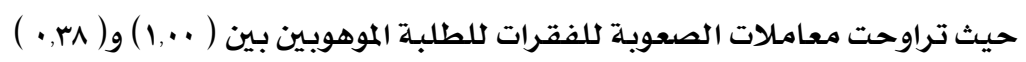

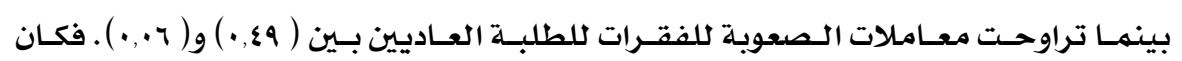

بذلك الاختلاف بمعامل صعوبة الفقرات جليا .

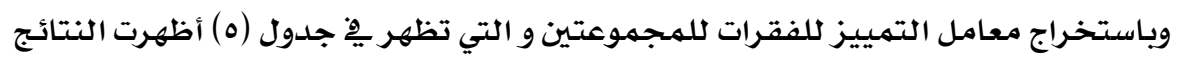

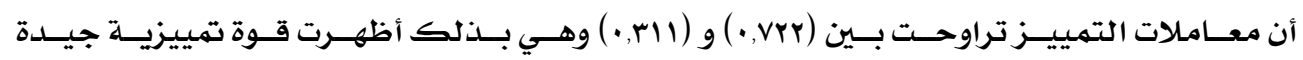
لأغراض البحث معاتل

وبإجراء تحليل التباين الأحادي المبين هِّ جدول (ج) للكثف عن قدرة الاختبـار على التمييز

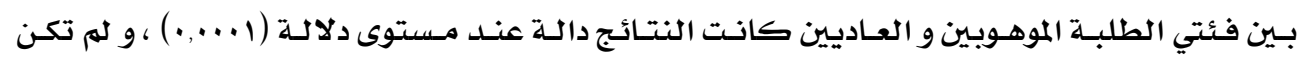

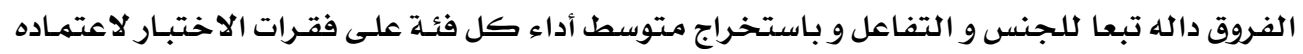

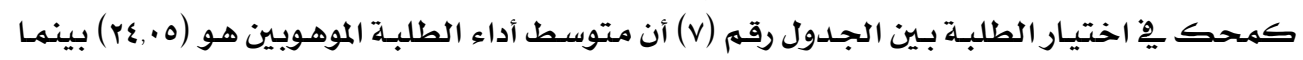

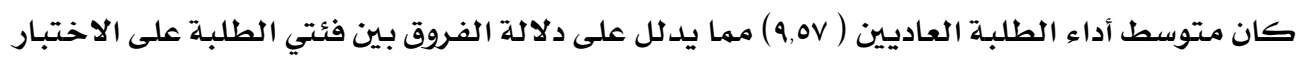

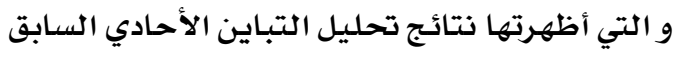

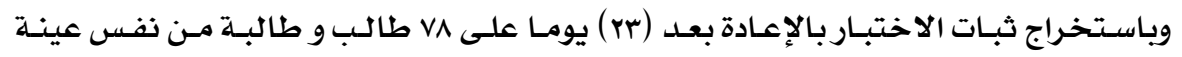

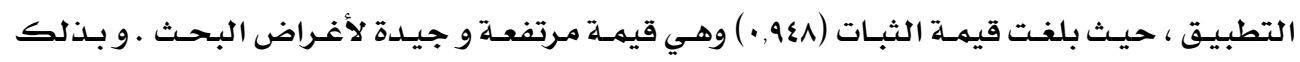

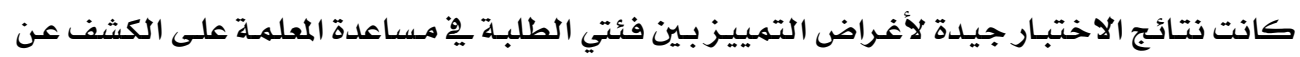

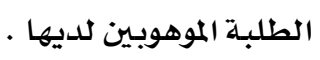


ا- أبو عميرة ، محبات، (1999) . المتفوقون و الرياضيات ، مكتبة الدار العربية ، القاهرة ، مصر.

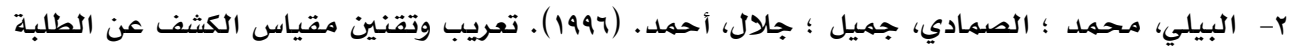

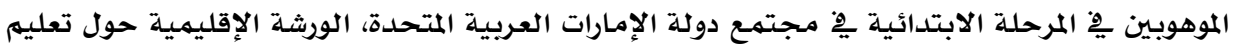

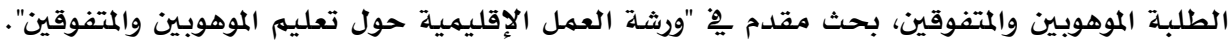
الألودن، عمّان. r- العمر ، بدر . ( •199) ، المتفوقون : تعريفهم ، رعايتهم ، برامجهم ، و إعداد مدرسيهم ، مجلة دراسات ، المجات

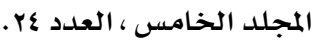

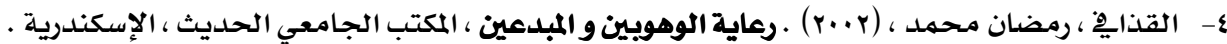

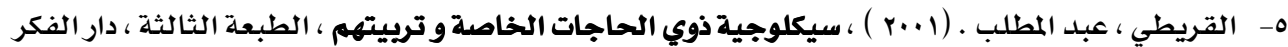
العربي ، القاهرة .

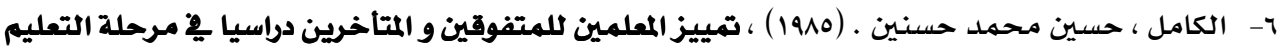

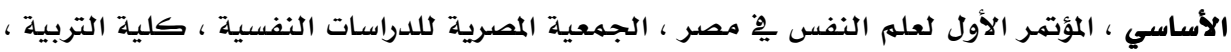

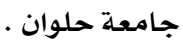
V- الكيلاني، عبد الله زيد ؛ جروان، فتحي. (199v). دليل اختبارات الاستعداد الأكاديمي، الأردن، عمّان:

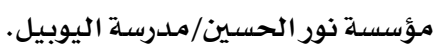

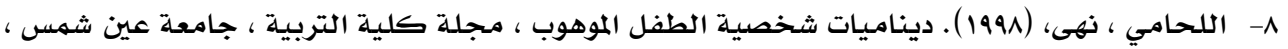

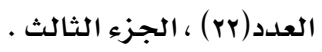

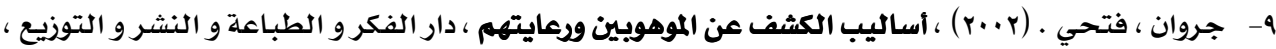

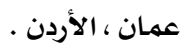

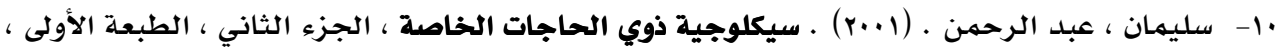

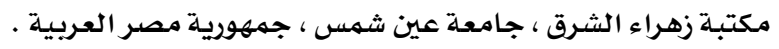

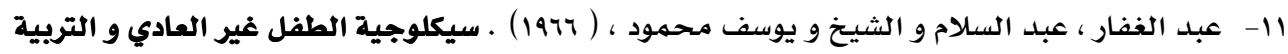

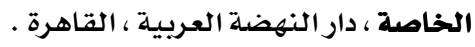

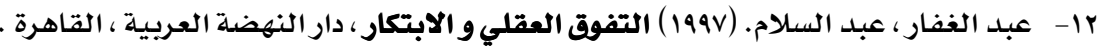

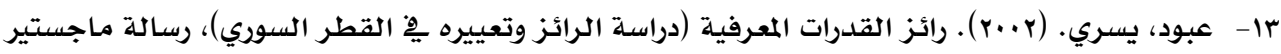
غير منشورة، الجمهورية العربية السورية، دمشق: جامعة دمشق.

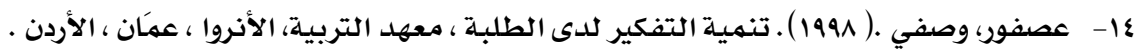

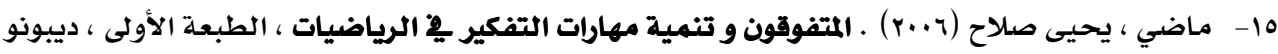

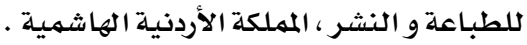




\section{المراجع الإنجليزية}

1- Berger, S. (1999). Student selection for gifted/talented programs: May's feature. The ERIC clearinghouse on disabilities and gifted education. Parent news archives for May-June. [On-line]. Available: http://ericae.net/testcol.htm

2- Clark, B.: Growing up Giftedness 4th Ed ., Macmillan Publishing Company, 1992

3- Corsmit,and Others. Information Processing in Intellectually Highly Gifted Children by Solving Mathematical Tasks, Gifted Education International ,Vol .(6) No.(3) . 1990.

4- Getzels, J.W. \& Jackson, p.w . Creativity and Intelligence, Explorations with Gifted Students NYC; Wiley 1962

5- Greenlaw, M.J. \& Mclontosh, M.E. Educational the gifted. Chicago: American Library Association, 1988.

6- June, M , Curriculum Development for the Gifted, Pro .Ed, Austen, Texas, 1982 .

7- Kitano .M . Intellectual Abilities and Psychological intensities in Young Gifted Children , Implications for the Gifted , Roper Review , No (13) ,1993 .

8- Wallace ,T . the Effects of Inrechment on Gifted Students , A Quantitative Synthesis D.A.I., Vol (50), No (9), 1990 .

9- Wilson , S . Effected Syntic Training on Gifted and No Gifted Kindergarten Student , Ph .D .Texas Woman's University , Summary ,D.A.I. ,Vol(3-5A), 1992 . 


\section{ABSTRACT}

The aim of the research is to answer about the following question :

what is effectiveness of mathematics ability test to distinguishing gift student.

For answering about the research question.

Thirdly; passing the test of mathematical ability, which was constructed out of the curriculum, and measured mathematical relationships between the numbers, which was trusted and constructed by four members of teaching committee, who, have the dectorate degree from Amman Arabic University for highest studies,. Thin they found out sememes of measurement validity by application on (63) students (male and female) from the intelligent students and (72) students (male and Female) from the normal students, After that Factor analysis is taking ,the results appeared that: the single dimension, which paraphrased one factor (34.294) from the whole variance; but the second factor paraphrased (5.991) from the expounder variance.

It is clear that one factor expounded (6-1) of the second factor which exposes the single dimension in testing. Then it founded out psychometry specification for the items, also, it found out mean parameter for items to each sample of the students as it shown.

The results paraphrased the differences of mean parameter of each item on the two samples:( normal and intelligent) students. Also, the result paraphrased that mean parameter for items for items for the intelligent students between (1.00) and (0.30) while the mean parameter for items reached (0.49) and (0.06) that there was the difference with the mean parameter for the items which was found.

By finding out distinguish parameter for items according to the tow groups. The results paraphrased that the distinguished parameters reached between (0.722) and (0.311) with this it achieved good, distinguishing power for the aims of the research.

By application of Anova for exposure about testing ability at distinguishing between the tow samples of the students (intelligent and normal).

The results were sematic at the level (0.0001), and by finding out the Arithmetic mean of the performance of each sample at the testing items to consider it as a standard. 
In choosing the students. It had shown that the arithmetic mean of performance of the intelligent students was (24.05), but the arithmetic mean of the performance of the normal students was (9.57) which shown that: the sematic differences between the tow samples of the students at testing which shown by the results of Anova. And by finding out the reliability of test by repetition after (23) days at (78) students (male and female) from the same sample of the application sample, so the reliability value reached (0.948), and It is good and high value for the aims of the research, so that the results of good test were for distinguishing aims between the two samples of the students in helping the teacher to expose the intelligent students according to her. 